\title{
Effect of Eating out on Quality and Quantity of Sleep among Japanese in Their 20s
}

\author{
Tomohide Yagyu1 ${ }^{1}$, Kohei Akazawa², Minami Ebihara², Junta Ishikawa², Ouchi Koichi² \\ ${ }^{1}$ Department of Health Informatics, Suzuka University of Medical Science, Suzuka, Japan \\ ${ }^{2}$ Department of Medical Informatics, Niigata University Medical \& Dental Hospital, Niigata, Japan \\ Email: yagyu.tomohide@gmail.com
}

How to cite this paper: Yagyu, T., Akazawa, K., Ebihara, M., Ishikawa, J. and Koichi, O. (2018) Effect of Eating out on Quality and Quantity of Sleep among Japanese in Their 20s. Health, 10, 1261-1269. https://doi.org/10.4236/health.2018.109097

Received: August 31, 2018

Accepted: September 24, 2018

Published: September 27, 2018

Copyright (c) 2018 by authors and Scientific Research Publishing Inc. This work is licensed under the Creative Commons Attribution International License (CC BY 4.0).

http://creativecommons.org/licenses/by/4.0/

\begin{abstract}
The purpose of this study was to analyze the effects of eating out or skipping meals on the quality or quantity of sleep in young Japanese adults. We analyzed 584 eligible subjects (283 men and 301 women) aged 20 - 29 years. Information on diet and individual lifestyle characteristics, including sleep quality, which was assessed by the Pittsburgh Sleep Quality Index Japanese version (PSQI-J), were obtained by participants completing an online questionnaire. Kendall's tau coefficient was used to measure the ordinal association between diet and sleep quality. The Mann-Whitney $U$ test was performed to evaluate differences in sleep quality by frequency of eating out and frequency of skipping meals. The results revealed a significant difference in sleep duration and PSQI-J score between people who eat out $>2$ times a week ("high restaurants group") and those who do not ("low restaurants group"). Furthermore, whereas the mean sleep duration in the "high restaurants group" was 6.59 hours, the mean sleep duration in the "low restaurants group" was 6.90 hours ( $\mathrm{p}=$ 0.007). The PSQI-J score was significantly higher in the "high restaurants group" than in the "low restaurants group" (4.91 versus $4.37, \mathrm{p}=0.006$ ). Significant differences were also evident in the test's component scores: C2, C3, and C7. No significant difference was found in sleep duration and PSQI-J score between the groups in terms of frequency of skipping meals (breakfast, lunch, and dinner). Our results suggested that excessive intake by eating out more than twice a week may lead to short sleep duration and high PSQI-J score.
\end{abstract}

\section{Keywords}

Sleep Quality, Dietary Habits, Cross-Sectional Study, Pittsburgh Sleep Quality Index, Eating out

\section{Introduction}

Although the functions of sleep are yet to be fully determined, it has been sug- 
gested that the most important ones are the conservation of energy and restoration of body functions [1]. Qualitatively good and sufficient sleep is crucial for performing several daily life activities [1]. However, the prevalence of short sleep duration has been increasing recently. The weighted prevalence of self-reported short sleep duration, defined as $\leq 6 \mathrm{~h}$ per day, among US civilian employed workers between 1985 and 1990, was 24.2\% [2]. Furthermore, in a combined sample obtained from 2004 to $2007,29.9 \%$ of workers reported short sleep duration [2].

Chen et al. highlight how young adults who sleep $<7 \mathrm{~h}$ overnight are more likely to report low health-related quality of life than those sleeping $\geq 7 \mathrm{~h}$ overnight [3]. Previous studies have suggested that people with insufficient sleep syndrome have significantly higher depression scores than healthy sleepers, and that sleep problems are strongly associated with suicidal ideation and behavior [4] [5] [6].

Yoshitaka et al. conducted a questionnaire survey completed by 4450 Japanese subjects aged 20 - 29 years. Up to $48.5 \%$ of participants (53.6\% males and $43.8 \%$ females) reported sleeping for $<7$ hours [7]. In Japan, among those aged 20 - 29 years, suicide is the most often reported cause of death [8]. It is therefore worth considering the effects of short sleep duration on mental health to ameliorate sleep behavior among young adults.

Skipping breakfast, and excessive intake of staple foods at lunch and dinner are known to be associated with poor sleep-wake regularity [1]. However, according to advanced research by Nguyen et al., fast-food and full-service restaurant consumption were associated with an increase in daily total energy intakes of $813.75 \mathrm{~kJ}(194.49 \mathrm{kcal})$ and $858.04 \mathrm{~kJ}(205.21 \mathrm{kcal})$, respectively [9].

With this knowledge, eating out as well as skipping meals may be factors associated with poor sleep behavior. The present study was conducted to analyze the effects of eating out or skipping meals on the quality or quantity of sleep in young Japanese adults.

\section{Materials and Methods}

\subsection{Study Design and Participants}

Participants were recruited from a database that is administered by a Japanese private company specializing in questionnaire research. An online survey that was developed by a survey company targeted individuals aged $20-29$ years without serious disease. Informed consent was obtained from all participants prior to enrollment. All 800 participants who accessed the survey web page responded to the questionnaire.

Exclusion criteria were: 1 ) spends excessive hours in bed ( $\geq 16 \mathrm{~h} /$ day $[n=67])$; 2) responded with invalid answers to questionnaire items (e.g., selecting the same number on every question $[\mathrm{n}=60]$ ); responded with answers that were inconsistent with those of other questions $(\mathrm{n}=19)$; and 3 ) takes any sleeping medication $(\mathrm{n}=60)$. Finally, 584 participants ( 283 men and 301 women) were analyzed in this study. 


\subsection{Questionnaire}

The questionnaire used in the present study consisted of three sections. Section 1 collected basic information on respondents, including age, sex, residential address, occupation, marriage status, and family structure. Section 2 comprised questions on diet, including questions on frequency of skipping meals (breakfast, lunch, and dinner) per week. The survey respondents were also asked to provide average frequencies of visiting limited-service restaurants and full-service restaurants per week. Section 3 examined sleep quality, which was assessed by the Pittsburgh Sleep Quality Index Japanese version (PSQI-J). The PSQI-J comprises self-rated items from which seven component scores (subscale scores ranging from 0 to 3 ) are generated: sleep quality (C1); sleep latency $(\mathrm{C} 2)$; sleep duration (C3); sleep efficiency (C4); sleep disturbance (C5); hypnotics medication use (C6); and daytime dysfunction (C7). The sum of these seven component scores yields one global score, i.e., subjective sleep quality (range, 0 - 21) [10].

\subsection{Statistical Analysis}

First, Fisher's exact test was performed to test the equality of distributions of sleeping times among the categories of age, sex, occupation, marriage status and number of residences.

Next, Kendall's tau coefficient was used to measure the ordinal association between dietary habits (i.e., frequency of visiting limited-service restaurants or full-service restaurants per week and frequency of skipping meals, i.e., breakfast, lunch, and dinner) and sleep quality (i.e., sleep duration, seven component scores and PSQI-J global score).

The participants were divided into two groups based on whether they skipped breakfast $\geq 3$ times per week versus $<3$ times per week. Differences in sleep quality among the two groups were analyzed by the Mann-Whitney U test. Similarly, the participants were divided into two groups according to weekly lunch or dinner frequency, and differences in sleep quality were evaluated by the Mann-Whitney U test.

The participants were also divided into two groups based on whether they used limited-service restaurants or full-service restaurants $\geq 2$ times per week ("high restaurants group") versus $<2$ times per week ("low restaurants group"). Differences in sleep quality between the two groups were analyzed by the Mann-Whitney U test.

The R software package (version 3.4.1, 2017, The R Foundation for Statistical Computing) was used for all statistical analyses. All $\mathrm{p}$ values are two-tailed and those less than 0.05 were considered statistically significant.

\section{Results}

The basic characteristics of the participants included this study are shown in Table 1. Of these, 102 young adult participants were students, and 192 adult participants were full-time employees. A significant difference was observed in sleep duration between young adults and adults $(\mathrm{p}=0.039)$.

For the results obtained from measuring the ordinal association between die- 
tary habits and sleep quality, all values for the correlation coefficient were $<0.15$.

The sleep qualities of each group with different frequencies in meal intake are shown in Table 2. No significant difference was found in sleep duration and PSQI-J score between the groups in terms of frequency of skipping meals (breakfast, lunch, and dinner).

Table 3 shows the differences in sleep quality between the "high restaurants group" and "low restaurants group". A significant difference was noted in mean sleep duration. Whereas the mean sleep duration in the "high restaurants group" was 6.59 hours, the mean sleep duration in the "low restaurants group" was 6.90 hours $(\mathrm{p}=0.007$ ). The PSQI-J score was significantly higher in the "high restaurants group" than in the "low restaurants group" (4.91 versus $4.37, \mathrm{p}=0.006$, Figure 1). Significant differences were evident in the component scores of $\mathrm{C} 2$ ("high restaurants group": 0.94, "low restaurants group": 0.84, p = 0.04), C3 ("high restaurants group": 1.25, "low restaurants group": 1.03, p =0.007), and C7 ("high restaurants group": 0.68, "low restaurants group": 0.56, p = 0.025) among the two groups (Figure 2).

Table 1. Baseline characteristics of the study subjects.

\begin{tabular}{|c|c|c|c|}
\hline & Young adults & Adults & p \\
\hline & $(20-25$ years $)$ & $(26-29$ years $)$ & $P$ ratus \\
\hline$n$ & 227 & 357 & \\
\hline \multicolumn{4}{|l|}{ Sex } \\
\hline Male & $113(49.8)$ & $170(47.6)$ & \\
\hline Female & $114(50.2)$ & $187(52.4)$ & 0.612 \\
\hline \multicolumn{4}{|l|}{ Occupation } \\
\hline Full-time employee & $66(29.1)$ & $192(53.8)$ & \\
\hline Part time employee & $28(12.3)$ & $52(14.6)$ & \\
\hline Self-employed & $5(2.2)$ & $11(3.1)$ & \\
\hline Homemaker & $14(6.2)$ & $54(15.1)$ & \\
\hline Student & $102(44.9)$ & $11(3.1)$ & \\
\hline Unemployed & $12(5.3)$ & $37(10.4)$ & $<0.001$ \\
\hline \multicolumn{4}{|l|}{ Marriage status } \\
\hline Married & $25(11.0)$ & $114(31.9)$ & \\
\hline Unmarried & $183(80.6)$ & $209(58.5)$ & \\
\hline Divorced or widowed & $19(8.4)$ & $34(9.5)$ & $<0.001$ \\
\hline \multicolumn{4}{|c|}{ Number of people living together } \\
\hline Alone & $81(35.7)$ & $86(24.1)$ & \\
\hline Two & $26(11.5)$ & $78(21.8)$ & \\
\hline Three or more & $120(52.9)$ & $193(54.1)$ & $<0.001$ \\
\hline \multicolumn{4}{|l|}{ Sleep duration (hours) } \\
\hline$<6$ & $41(18.1)$ & $75(21.0)$ & \\
\hline 6 to $<7$ & $51(22.5)$ & $112(31.4)$ & \\
\hline 7 to $<8$ & $79(34.8)$ & $101(28.3)$ & \\
\hline$\geq 8$ & $56(24.7)$ & $69(19.3)$ & 0.039 \\
\hline
\end{tabular}

): Percentages. Due to rounding, percentages may not total $100 \%$. p-values determined by using Fisher's exact test. 
Table 2. Sleep quality of each group with different frequencies in skipping of meals (mean \pm standard deviation).

\begin{tabular}{|c|c|c|c|c|c|c|c|c|c|}
\hline & \multicolumn{3}{|c|}{$\begin{array}{c}\text { Number of times breakfast } \\
\text { was skipped }\end{array}$} & \multicolumn{2}{|c|}{$\begin{array}{c}\text { Number of times lunch } \\
\text { was skipped }\end{array}$} & \multicolumn{4}{|c|}{$\begin{array}{c}\text { Number of times dinner } \\
\text { was skipped }\end{array}$} \\
\hline & $\begin{array}{l}\geq 3 \text { times } \\
\mathrm{n}=161\end{array}$ & $\begin{array}{l}<3 \text { times } \\
\mathrm{n}=423\end{array}$ & $\mathrm{p}$-value & $\begin{array}{l}\geq 3 \text { times } \\
\mathrm{n}=65\end{array}$ & $\begin{array}{l}<3 \text { times } \\
\mathrm{n}=519\end{array}$ & $\mathrm{p}$-value & $\begin{array}{l}\geq 3 \text { times } \\
\mathrm{n}=24\end{array}$ & $\begin{array}{l}<3 \text { times } \\
\mathrm{n}=560\end{array}$ & $p$-value \\
\hline Sleep duration & $6.76 \pm 1.55$ & $6.66 \pm 1.26$ & 0.416 & $6.49 \pm 1.59$ & $6.71 \pm 1.31$ & 0.42 & $6.21 \pm 1.37$ & $6.71 \pm 1.34$ & 0.087 \\
\hline \multicolumn{10}{|l|}{ PSQI-J } \\
\hline Global score & $4.88 \pm 2.85$ & $4.68 \pm 2.61$ & 0.43 & $4.91 \pm 2.95$ & $4.72 \pm 2.64$ & 0.761 & $5.00 \pm 3.06$ & $4.73 \pm 2.66$ & 0.784 \\
\hline \multicolumn{10}{|l|}{ Component scores } \\
\hline $\begin{array}{l}\text { Sleep quality } \\
\text { (C1) }\end{array}$ & $1.43 \pm 0.80$ & $1.38 \pm 0.74$ & 0.366 & $1.43 \pm 0.73$ & $1.39 \pm 0.76$ & 0.451 & $1.54 \pm 0.83$ & $1.38 \pm 0.76$ & 0.33 \\
\hline $\begin{array}{l}\text { Sleep latency } \\
\text { (C2) }\end{array}$ & $0.99 \pm 0.90$ & $0.86 \pm 0.84$ & 0.159 & $0.95 \pm 0.94$ & $0.89 \pm 0.85$ & 0.791 & $0.96 \pm 0.86$ & $0.89 \pm 0.86$ & 0.651 \\
\hline $\begin{array}{c}\text { Sleep duration } \\
\text { (C3) }\end{array}$ & $1.12 \pm 0.99$ & $1.20 \pm 0.86$ & 0.308 & $1.26 \pm 1.02$ & $1.17 \pm 0.88$ & 0.487 & $1.38 \pm 1.01$ & $1.17 \pm 0.89$ & 0.249 \\
\hline $\begin{array}{l}\text { Sleep efficiency } \\
\text { (C4) }\end{array}$ & $0.29 \pm 0.69$ & $0.31 \pm 0.71$ & 0.981 & $0.37 \pm 0.84$ & $0.29 \pm 0.69$ & 0.799 & $0.46 \pm 0.93$ & $0.30 \pm 0.69$ & 0.433 \\
\hline Sleep disturbance (C5) & $0.32 \pm 0.50$ & $0.33 \pm 0.50$ & 0.976 & $0.32 \pm 0.50$ & $0.33 \pm 0.50$ & 0.958 & $0.21 \pm 0.41$ & $0.33 \pm 0.50$ & 0.255 \\
\hline Daytime dysfunction (C7) & $0.72 \pm 0.82$ & $0.61 \pm 0.76$ & 0.157 & $0.57 \pm 0.73$ & $0.65 \pm 0.79$ & 0.468 & $0.46 \pm 0.66$ & $0.65 \pm 0.78$ & 0.247 \\
\hline
\end{tabular}

p-values determined via the Mann-Whitney U test.

Table 3. Differences in sleep quality between the "low restaurants group" and "high restaurants group" (mean \pm standard deviation).

\begin{tabular}{|c|c|c|c|}
\hline & Low restaurants group & High restaurants group & \\
\hline & $\mathrm{n}=185$ & $\mathrm{n}=399$ & $\mathrm{p}$-value \\
\hline Sleep duration & $6.90 \pm 1.40$ & $6.59 \pm 1.31$ & 0.007 \\
\hline \multicolumn{4}{|l|}{$P S Q I-J$} \\
\hline Global score & $4.37 \pm 2.76$ & $4.91 \pm 2.61$ & 0.006 \\
\hline \multicolumn{4}{|l|}{ Component scores } \\
\hline Sleep quality (C1) & $1.37 \pm 0.77$ & $1.40 \pm 0.76$ & 0.543 \\
\hline Sleep latency $(\mathrm{C} 2)$ & $0.80 \pm 0.84$ & $0.94 \pm 0.86$ & 0.04 \\
\hline Sleep efficiency (C4) & $0.28 \pm 0.69$ & $0.32 \pm 0.71$ & 0.37 \\
\hline Sleep disturbance (C5) & $0.34 \pm 0.54$ & $0.32 \pm 0.48$ & 0.99 \\
\hline Daytime dysfunction (C7) & $0.56 \pm 0.79$ & $0.68 \pm 0.77$ & 0.025 \\
\hline
\end{tabular}

p-value determined via the Mann-Whitney U test. High restaurants group: who used limited-service restaurants or full-service restaurants $\geq 2$ times per week. Low restaurants group: who used limited-service restaurants or full-service restaurants $<2$ times per week. 

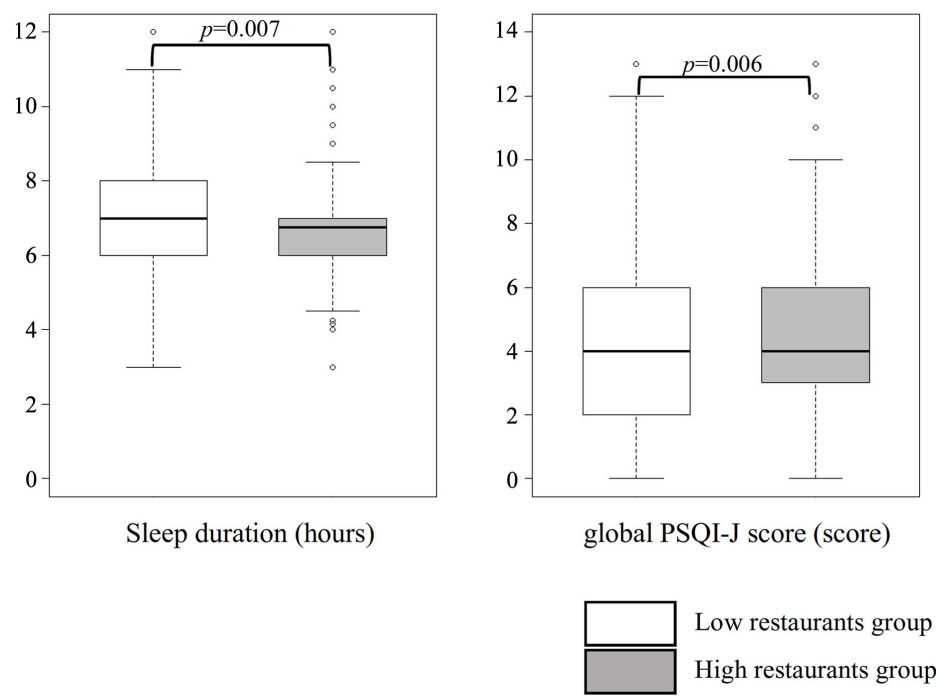

Figure 1. Differences in sleep duration and global score of the PSQI-J between the "low restaurants group" and "high restaurants group".

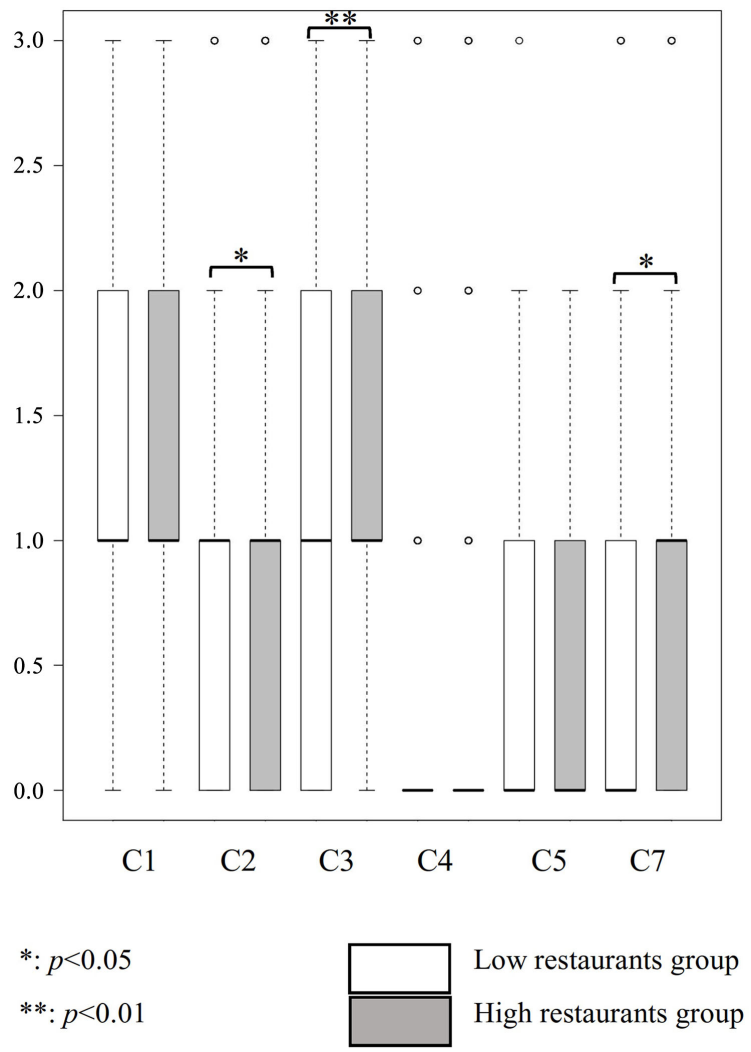

Figure 2. Differences in component PSQI-J scores between the "low restaurants group" and "high restaurants group".

\section{Discussion}

In this study, the relationship between dietary habits and quality/quantity of sleep was quantitatively evaluated based on a questionnaire survey. A significant difference was observed in sleep duration and PSQI-J score between people who 
eat out $>2$ times a week and those who do not. Binh T. Nguyen et al. showed that both fast-food and full-service restaurant food consumption among adults were associated with significant increases in energy, sugar, and saturated fat [9]. Moreover, they showed that the effects of fast-food restaurant consumption were significantly larger for young (aged 20 - 34 years) compared with older adults. Further, according to other research, excessive intake of staple foods at lunch and dinner may be associated with poor sleep-wake regularity [1]. Given the findings of these previous studies and those of the current study, we considered excessive intake by eating out more than twice a week may lead to short sleep duration and high PSQI score.

A significant difference was found in PSQI-J component score C7 (daytime dysfunction) between people who eat out $>2$ times a week and those who do not. The component score is derived from the question, "During the past month, how often have you had trouble staying awake while driving, eating meals, or engaging in social activity?" Kessler et al. showed that insomnia has a significant association with non-workplace injuries, although this association was weaker than that with workplace injuries [11]. Based on this evidence, we considered that eating out more than twice a week may influence not only mental health but also physical health.

The lifestyle of people in their 20s changes greatly because they typically move from studying and graduating from vocational schools and universities to fulltime employment. Under such circumstances, it is easy to imagine that the frequency of eating out, which is relatively easy to do, is high. Eating out could be due to having less time in the day to prepare meals, coinciding with increased stress and anxiety. This in turn also impacts sleep quality. However, our results showed that frequently eating out may reduce quantity and quality of sleep. We think that it is necessary for young people to receive education on, not only the importance of cooking their own meals, but also the actual skills required for cooking one's own meals. It is also necessary to pay attention to dietary habits so that daily total energy intake is not increased by eating out. In this study, for those aged over 26 years, more than half slept only $<7$ hours. Therefore, similar education is also required as health guidance in companies.

The strength of this study lies in the fact that we analyzed the effects of eating out or skipping meals on quality and quantity of sleep. However, this study also has several limitations. First, due to the nature of the cross-sectional study design, causal relationships between dietary habits and sleep quality should be interpreted with caution. Second, information on dietary habits and sleep quality was subjective, and can be considered as lacking objectivity. Further interventional studies by objective assessment of skipping meals or eating out and sleep quality will be needed.

\section{Conclusion}

The present study was conducted to analyze the effects of eating out or skipping 
meals on the quality or quantity of sleep in young Japanese adults. No significant difference was found in sleep duration and PSQI-J score between people who skip breakfast $\geq 3$ times per week and those who do not. Similarly, there were no significant differences in sleep duration and PSQI-J score by lunch or dinner frequency. A significant difference was observed in sleep duration and PSQI-J score between people who eat out $>2$ times a week and those who do not. Our results suggested that excessive intake by eating out more than twice a week may lead to short sleep duration and high PSQI-J score.

\section{Conflicts of Interest}

The authors declare no conflicts of interest regarding the publication of this paper.

\section{References}

[1] Yamaguchi, M., Uemura, H., Katsuura-Kamano, S., Nakamoto, M., Hiyoshi, M., Takami, H., Sawachika, F., Juta, T. and Arisawa, K. (2013) Relationship of Dietary Factors and Habits with Sleep-Wake Regularity. Asia Pacific Journal of Clinical Nutrition, 22, 457-465.

[2] Luckhaupt, S.E., Tak, S. and Calvert, G.M. (2010) The Prevalence of Short Sleep Duration by Industry and Occupation in the National Health Interview Survey. Sleep, 33, 149-159. https://doi.org/10.1093/sleep/33.2.149

[3] Chen, X., Gelaye, B. and Williams, M.A. (2014) Sleep Characteristics and Health-Related Quality of Life among a National Sample of American Young Adults: Assessment of Possible Health Disparities. Quality of Life Research, 23, 615-627. https://doi.org/10.1007/s11136-013-0475-9

[4] Morita, Y., Sasai-Sakuma, T., Asaoka, S. and Inoue, Y. (2015) Prevalence and Correlates of Insufficient Sleep Syndrome in Japanese Young Adults: A Web-Based Cross-Sectional Study. Journal of Clinical Sleep Medicine, 11, 1163-1169. https://doi.org/10.5664/jcsm.5092

[5] McDonald, K.C., Saunders, K. and Geddes, J.R. (2017) Sleep Problems and Suicide Associated with Mood Instability in the Adult Psychiatric Morbidity Survey, 2007. Australian \& New Zealand journal of Psychiatry, 51, 822-828. https://doi.org/10.1177/0004867416687398

[6] Chu, C., Hom, M.A., Rogers, M.L., Stanley, I.H., Ringer-Moberg, F.B., Podlogar, M.C., Hirsch, J.K. and Joiner, T.E. (2017) Insomnia and Suicide-Related Behaviors: A Multi-Study Investigation of Thwarted Belongingness as a Distinct Explanatory Factor. Journal of Affective Disorders, 208, 153-162. https://doi.org/10.1016/j.jad.2016.08.065

[7] Kaneita, Y., Ohida, T., Uchiyama, M., Takemura, S., Kawahara, K., Yokoyama, E., Miyake, T., Harano, S., Suzuki, K., Yagi, Y., Kaneko, A., Tsutsui, T. and Akashiba, T. (2005) Excessive Daytime Sleepiness among the Japanese General Population. Journal of Epidemiology, 15, 1-8. https://doi.org/10.2188/jea.15.1

[8] Ministry of Health Labour and Welfare (2016) Deaths by Causes (The Condensed List of Causes of Death for Japan), Sex and Age: Japan.

https://www.e-stat.go.jp/en/stat-search/files?page=3\&query=Deaths $\% 20$ by $\% 20$ cause s\&sort=year_month\%20desc\&layout=dataset\&stat_infid=000031450320

[9] Nguyen, B.T. and Powell, L.M. (2014) The Impact of Restaurant Consumption 
among US Adults: Effects on Energy and Nutrient Intakes. Public Health Nutrition, 17, 2445-2452. https://doi.org/10.1017/S1368980014001153

[10] Doi, Y., Minowa, M., Uchiyama, M., Okawa, M., Kim, K., Shibui, K. and Kamei, Y. (2000) Psychometric Assessment of Subjective Sleep Quality Using the Japanese Version of the Pittsburgh Sleep Quality Index (PSQI-J) in Psychiatric Disordered and Control Subjects. Psychiatry Research, 97, 165-172.

https://doi.org/10.1016/S0165-1781(00)00232-8

[11] Kessler, R.C., Berglund, P.A., Coulouvrat, C., Fitzgerald, T., Hajak, G., Roth, T., Shahly, V., Shillington, A.C., Stephenson, J.J. and Walsh, J.K. (2012) Insomnia, Comorbidity, and Risk of Injury among Insured Americans: Results from the America Insomnia Survey. Sleep, 35, 825-834. https://doi.org/10.5665/sleep.1884 